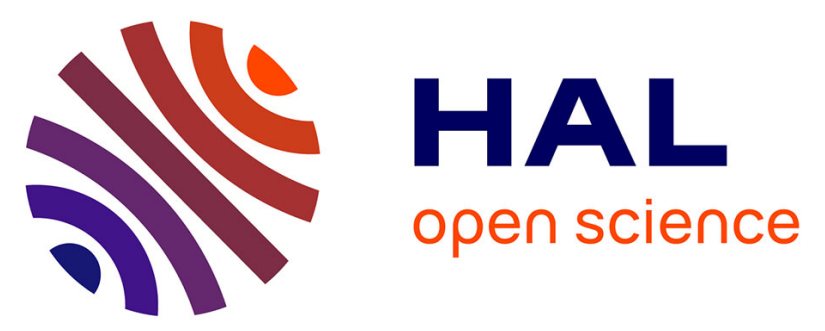

\title{
Visible-NIR Photodetectors Based on Low-Dimensional GeSe Micro-Crystals Designed Morphology and Improved Photoresponsivity
}

F. Zhao, X. Luo, L. Liu, J. Lv, X. Fan, X. Qiao, Y. Xu, L. Kienle, Xianghua Zhang, G. Qian

\section{To cite this version:}

F. Zhao, X. Luo, L. Liu, J. Lv, X. Fan, et al.. Visible-NIR Photodetectors Based on Low-Dimensional GeSe Micro-Crystals Designed Morphology and Improved Photoresponsivity. ChemPhysChem, 2020, 21 (5), pp.397-405. 10.1002/cphc.201901217 . hal-02536600

\section{HAL Id: hal-02536600 https://hal-univ-rennes1.archives-ouvertes.fr/hal-02536600}

Submitted on 14 Apr 2020

HAL is a multi-disciplinary open access archive for the deposit and dissemination of scientific research documents, whether they are published or not. The documents may come from teaching and research institutions in France or abroad, or from public or private research centers.
L'archive ouverte pluridisciplinaire HAL, est destinée au dépôt et à la diffusion de documents scientifiques de niveau recherche, publiés ou non, émanant des établissements d'enseignement et de recherche français ou étrangers, des laboratoires publics ou privés. 
DOI: 10.1002/ ((please add manuscript number))

Article type: Full Paper

\section{Visible-NIR Photodetectors Based on Low Dimensional GeSe Micro-crystals: Designed Morphology and Improved Photoresponsivity}

Feiyu Zhao, ${ }^{a}$ Xue Luo, ${ }^{a}$ Lixiang Liu, ${ }^{b}$ Jianhang Lv, ${ }^{b}$ Xianping Fan, ${ }^{a}{ }^{*}$ Xvsheng Qiao, ${ }^{a}$ * Yang $X u,{ }^{a, b^{*}}$ Lorenz Kienle, ${ }^{c}$ Xianghua Zhang, ${ }^{d}$ Guodong Qian ${ }^{a}$

Mr. F. Y. Zhao, Mrs. X. Luo, Dr. X. S. Qiao, Prof. Y. Xu, Prof. G.D. Qian, Prof. X. P. Fan State Key Laboratory of Silicon Materials \& School of Materials Science and Engineering Zhejiang University

Hangzhou 310027, P. R. China

Email: qiaoxus@zju.edu.cn; fanxp@zju.edu.cn; yangxu-isee@zju.edu.cn

L. X. Liu, J. H. Lv, Prof. Y. Xu

School of Information Science and Electronic Engineering

Zhejiang University

Hangzhou 310027, P. R. China

Prof. L. Kienle

Institute for Material Science, Synthesis and Real Structure

Christian Albrechts University Kiel

Kaiserstr. 2, 24143 Kiel, Germany

Prof. X. H. Zhang

Laboratory of Glasses and Ceramics, Institute of Chemistry

CNRS-Université de Rennes I

Campus de Beaulieu, 35042 Rennes cedex, France

*Corresponding author

Keywords: GeSe; photodetectors; micro-sheet; micro-belt; photoresponsivity

Abstract: The GeSe micro-sheets and GeSe micro-belts have been synthesized by a facile onepot wet chemical method in 1-octadecene solvent and oleic acid solvent, respectively. The larger adsorption of oleic acid molecules on the (002) plane promoted the growth along [010] direction of the GeSe micro-belts and limited carrier transport in one direction along [010], which resulted in higher carrier concentration and mobility of the GeSe micro-belts. The photodetection performance of the photodetectors based on the single GeSe micro-sheet and the single GeSe micro-belt was investigated under illumination of $532 \mathrm{~nm}, 980 \mathrm{~nm}$ and 1319 $\mathrm{nm}$ laser source for light illumination. Both photodetectors based on the single GeSe micro- 
sheet and the single GeSe micro-belt presented a high photoresponse, short response/recovery times and long-term durability. Moreover, the photodetector based on the single GeSe microbelt displays a broadband response with a high responsivity $(5562 \mathrm{~A} / \mathrm{W}$ at $532 \mathrm{~nm}, 1546 \mathrm{~A} / \mathrm{W}$ at $980 \mathrm{~nm})$ and detectivity $\left(3.01 \times 10^{12}\right.$ Jones at $532 \mathrm{~nm}, 8.38 \times 10^{11}$ Jones at $\left.980 \mathrm{~nm}\right)$. These excellent performances make the photodetector based on the single GeSe micro-belt very interesting as highly efficient photodetectors, especially in NIR region.

\section{Introduction}

Low-dimensional materials, especially one-dimensional (1D) and two-dimensional (2D) materials, with their unique properties have attracted a lot of attention from scientists across various disciplines for many decades. Discovery of carbon nanotubes in $1991^{[1]}$ have opened the door to 1D materials, such as micro- or nano- rods, wires, belts and tubes, which have significantly different properties from their bulk counterparts. For example, 1D materials have a lower melting point than corresponding bulk materials. ${ }^{[2]}$ Similarly, metal nanowires exhibited semiconductor or insulator properties in small size. ${ }^{[3,4]}$ Photodetectors based on semiconductor nanowires have high responsivity and sensitive switching characteristics. ${ }^{[5]}$ The photodetectors based on $\mathrm{ZnO}$ nanowire exhibited a high internal photoconductive gain as high as $10^{8}{ }^{[6]}$ Since the discovery of graphene in 2004 , 2D layered materials with atomic layer thickness have become hotspots in frontier research due to their unique dimensional characteristics. 2D material layers are connected by strong chemical bonds within, and the layers are connected by weak van der Waals forces, and can be easily peeled off into single or few layers. ${ }^{[7]}$ Graphene, ${ }^{[8,9]}$ with the electron mobility up to $10^{6} \mathrm{~cm}^{2} \cdot \mathrm{V}^{-1} \cdot \mathrm{s}^{-1}$, is a typical representative of $2 \mathrm{D}$ materials. But lack of the bandgap limits its application for high performance photodetector. Consequently, recent research focused on new alternative 2D semiconductors. Some transition metal dichalcogenides (TMD), such as $\mathrm{MoS}_{2}$, were reported to have high photosensitive and on/off ratio reach to $10^{8} \cdot{ }^{[10]}$ But their carrier mobility is still 
very low $\left(100 \mathrm{~cm}^{2} \cdot \mathrm{V}^{-1} \cdot \mathrm{s}^{-1}\right)$ compared with grapheme. The indirect bandgap of $1.29 \mathrm{eV}$ (bulk) and $1.9 \mathrm{eV}$ (monolayer) also limits their application in NIR photodetector. ${ }^{[1]}$ Black phosphorus (BP), owning to its narrower bandgap $(0.3 \mathrm{eV})$ and ultra-high electron mobility (10000-50000 $\left.\mathrm{cm}^{2} \cdot \mathrm{V}^{-1} \cdot \mathrm{s}^{-1}\right)$, has emerged as an exciting alternate for IR detection. ${ }^{[12,13]}$ However, it is not stable in ambient which seriously limits its processing and applications. ${ }^{[14]}$

GeSe, with a packed layer structure similar to BP, has bandgap of 1.1-1.2 eV which covers the solar light wavelength. ${ }^{[15]}$ Its hall mobility is $\sim 120 \mathrm{~cm}^{2} \cdot \mathrm{V}^{-1} \cdot \mathrm{s}^{-1}$ and carrier concentration is $\sim 5.31 \times 10^{16} \mathrm{~cm}^{-3}$ at low temperature. ${ }^{[16]}$ Compared with BP, the biggest merit of GeSe is its chemical inertness, e.g. its stability in ambient. Moreover, the bandgap can be tuned by changing the thickness of GeSe nanosheet. ${ }^{[17]}$ In previous reports ${ }^{[18-20]}$, 2D GeSe-based photodetectors exhibited high responsivity up to $3.5 \mathrm{~A} / \mathrm{W}$ at $808 \mathrm{~nm}^{[18]}, 870 \mathrm{~A} / \mathrm{W}$ at $405 \mathrm{~nm}$ ${ }^{[19]}$, and $2304 \mu \mathrm{A} / \mathrm{W}$ at $350 \mathrm{~nm}{ }^{[20]}$. Some research was also focused on $2 \mathrm{D}$ GeSe-based phototransistors, which exhibited ultrahigh responsivity up to or $1.6 \times 10^{5} \mathrm{~A} / \mathrm{W}$, but with long response and decay time $(0.28 \mathrm{~s}$ and $0.51 \mathrm{~s}) .{ }^{[21]}$ This is because gate voltage of the phototransistor was favorite to a high responsivity, but the concentration of defects and impurities were still not low enough to get a fast photoresponse. Some micro-size 1D GeSe belts or tubes and 2D GeSe sheets have been synthesized by different solution synthesis methods. $^{[22-26]}$ Xue et al. synthesized colloidal nanosheets by heating $\mathrm{GeI}_{4}$, diphenyl diselenide and hexadecylamine mixture and discovered its anisotropic photoresponse properties. ${ }^{[23]}$ Dimitri D et al. reported a solution-based seedless growth strategy and the single crystal colloidal GeSe nanobelts which were synthesized by hot-inject method with $\mathrm{GeI}_{4}$ and TOP-Se. ${ }^{[25]}$ Ye et al. reported the synthesis of the bulk GeSe by heating Se and Ge together under vacuum conditions, and then GeSe nanosheets were separated by direct sonication-assisted liquid phase exfoliation (LPE) from the bulk GeSe. ${ }^{[17]}$ However, the facile wet chemical synthesis method of low dimension structure GeSe and their application in the photodetector devices is rarely reported. In this paper, we report a one-pot wet chemical 
synthesis method for the direct synthesis of the GeSe micro-sheets and the GeSe micro-belts. Moreover, we fabricated photodetectors formed by single GeSe micro-sheet and GeSe microbelt, which demonstrated a remarkable responsivity and detectivity at $532 \mathrm{~nm}, 980 \mathrm{~nm}$ and $1319 \mathrm{~nm}$. Those photodetectors consisted of single GeSe micro-sheet or GeSe micro-belt showed very interesting features as highly efficient photodetectors.

\section{Results and Discussion}

Figure 1 shows the XRD patterns of the GeSe micro-sheets which were synthesized in 1octadecene solvent and the GeSe micro-belts which were synthesized in oleic acid solvent. Both XRD patterns exhibit prominent peaks in agreement with the JCPDS standard card (481226) of the orthorhombic structure. However, they present a stronger (400) peak compared with the bulk material (as shown in the standard card). For micro-sheets, the (400) peak intensity is substantially equal to the (111) peak. Oriented growth of micro-sheets along [010] and [001] directions promoted (400) crystal plane enlargement. For micro-belts, the (400) peak intensity is about $80 \%$ of (111) peak. Oriented growth of micro-belts along [001] direction also promoted (400) crystal plane enlargement, but the degree of enlargement is not as pronounced as for the micro-sheets.

The SEM image (Figure 2a) of the GeSe micro-sheets which were synthesized in 1octadecene solvent shows a rectangular slab shape with the geometry of about $20 \mu \mathrm{m} \times 15$ $\mu \mathrm{m} \times 500 \mathrm{~nm}$. Some step-like surface on the micro-sheet surface indicated a layered structure of the materials. In contrast, the sample which was synthesized in oleic acid exhibited beltshaped structure with the dimensions of about $20 \mu \mathrm{m} \times 500 \mathrm{~nm}$ (Figure $2 \mathrm{~d}$ ). The selected electron diffraction (SEAD) patterns of both GeSe micro-sheets (Figure 2b) and GeSe microbelts (Figure 2e) are highly similar and are identified as orthorhombic GeSe with Pnma space group. Insets in Figure $2 \mathrm{~b}$ and e are TEM images of single micro-sheet and micro-belt. From 
the SEAD patterns, the (020), (011) and (002) planes could be recognized with inter-planar spacings (d-spacings) of $0.191 \mathrm{~nm}, 0.290 \mathrm{~nm}$ and $0.218 \mathrm{~nm}$, respectively. These values also match well with the JCPDS standard card (No.48-1226). HRTEM images show (Figure 2c and f) that some $\{011\}$ lattice fringes are clearly imaged with the d-spacing of $0.290 \mathrm{~nm}$. For the micro-belt, according to the TEM image of the micro-belt (inset in Figure 2e) and the arrangement direction of the (020) crystal plane in the HRTEM (Figure 2f), the micro-belt exhibits an obvious oriented growth along the [010] direction.

Figure 3 illustrates the formation mechanism of GeSe micro-sheets and micro-belts. Ligand adsorption of the solvent on GeSe surfaces will influence the preferential growth orientation of GeSe crystals. GeSe is a typical layered structure with very saturated and stable layers, where there are no additional dangling bonds between layers. When the 1-octadecene was used as solvent, it did not produce chemisorption on the surface of the GeSe. As a consequence, the GeSe in 1-octadecene can grow into micro-sheet along [001] and [010] directions (Figure 3a). To further study their growth mechanism, We systematically observed the micro-crystal morphology (Figure 4) and Fourier Transform infrared (FTIR) spectra (Figure S1, Supporting Information) of GeSe synthesized with different oleic acid/1octadecene ratios $(0: 100 ; 20: 80 ; 40: 60 ; 60: 40 ; 80: 20 ; 100: 0)$. When the ratio of oleic acid/ 1-octadecene is 0: 100 (Figure 4a), most of the products are sheet-like structures with little difference between length and width. As the content of oleic acid gradually increases, the obtained GeSe micro-crystals become longer and narrower. When the oleic acid is introduced above $80 \%$, the obtained GeSe is basically a micro-belt structure (Figure $4 \mathrm{e}$ and $\mathrm{f}$ ). It is obvious that the morphology of the GeSe can undergo an alteration from micro-sheets to micro-belts by changing the ratio of oleic acid/1-octadecene. From the FTIR spectra (Figure S1a and b), it easily verifies the adsorption of oleic acid on the surface of the micro-belt, where the $1389 \mathrm{~cm}^{-1}$ peak assigned to $\mathrm{COO}^{-}$group ${ }^{[27]}$ (for oleic acid) only appeared in the micro-belt. With the increase of oleic acid content, the peak of $\mathrm{COO}^{-}$becomes more obvious. 
This is because the oleic acid which has a $-\mathrm{COOH}$ combines with Ge ions on the surface of GeSe to form a Ge-OCO bond. As a result, the oleic acid can be easily absorbed in the (002) planes in which GeSe chemically grow only along [010] direction, resulting in formation of GeSe micro-belts along the [010] direction (Figure 3b). This phenomenon confirmed that adhesion of oleic acid to (002) planes of GeSe promoted an orientational growth along [010] direction which resulted in the formation of the micro-belts.

The GeSe micro-sheets and the GeSe micro-belts exhibited diverse bandgaps owing to their different microstructure and thickness. When the layer numbers of the GeSe were more than 3, the transition from direct bandgap to indirect bandgap occurred, ${ }^{[28]}$ and the bandgap also changes with the thickness variation. In this work, the thickness of micro-sheet is about 152.1 $\mathrm{nm}$, while that of one micro-belt is about $82.9 \mathrm{~nm}$ (Figure S2). Figure 5a and b show the UVVis-NIR reflectance spectra of the GeSe micro-sheets and the GeSe micro-belts, respectively. Bandgap of the micro-sheet and the micro-belt were then determined by using Kubelka-Munk transformation from the reflectance spectra, where $x$ axis is $h v$ and y axis is $[\mathrm{F}(\mathrm{R}) h v]^{1 / 2}$. After linear extrapolation, the indirect bandgap of the GeSe micro-sheets and the GeSe micro-belts was found to be $1.02 \mathrm{eV}$ and $0.94 \mathrm{eV}$, respectively (Figure 5c and d). Those bandgaps of the micro-sheets and the micro-belts indicated their good photodetection application prospects in both visible and NIR region.

Table S1 presents the electrical properties of the synthesized GeSe micro-sheets and microbelts which are roughly estimated by Van der Pauw method. In results, GeSe micro-belts film exhibit much higher Hall mobility $\left(31.2 \mathrm{~cm}^{2} \cdot \mathrm{V}^{-1} \cdot \mathrm{s}^{-1}\right)$ and carrier concentration $\left(1.601 \times 10^{14}\right.$ $\mathrm{cm}^{-2}$ ) in comparison with the GeSe micro-sheets film, because the carrier transport was easily confined in quasi-one-dimension along [010] in the GeSe micro-belts. The GeSe micro-belts showed the much thinner perpendicular to the preferred growth direction (Figure $2 \mathrm{~d}$ and $\mathrm{c}$ ). An infinite potential well perpendicular to the growth direction was established, and the carrier mobility along the growth direction was much higher compared to perpendicular 
direction. In contrast, the GeSe micro-sheets exhibited no obvious quantum confinement effect, as the sizes along the three fundamental directions was comparable.

Through applying the van der Pauw method on the compressed powder film samples, the electrical properties of micro-sheets and micro-belts were not indeed evaluated precisely. This is because the scattering in grain boundary and a carrier transportation in sheet are quite different from single crystal. However, the purpose of our van der Pauw method is not to get specific values, but to compare the properties of GeSe micro-sheets and micro-belts. It can be proven that the micro-belt does have an improvement on electric property over the microsheet. Thus, the van der Pauw results are still relatively applicable for such purpose. In fact, the actual level of the micro-sheet should be around $70 \mathrm{~cm}^{2} / \mathrm{Vs}^{[16]}$ as previous research on bulk single crystal GeSe. The actual carrier mobility level of the micro-belt should be around $120 \mathrm{~cm}^{2} / \mathrm{Vs}^{[29]}$ It is similar with a GeSe single crystal film as previously reported and higher than that of the micro-sheet. Table S2 summarized previous study of the carrier mobility of materials by first-principles calculation, field effect transistor characterization and van der Pauw method.

The GeSe single micro-sheet or the single GeSe micro-belt was transferred on a $\mathrm{Si} / \mathrm{SiO}_{2}$ substrate with Au electrodes to fabricate photoconductive photodetector device (Figure 5a and b), respectively. Figure $6 \mathrm{c}$ and $\mathrm{d}$ show the I-V curves of the photodetectors based on the single GeSe micro-sheet and the single GeSe micro-belt under dark, and illumination with different light intensities using $980 \mathrm{~nm}$ laser as illumination sources. The electrical conductivity of the photodetectors based on the single GeSe micro-sheet $(1.17 \mu \mathrm{A})$ and the single GeSe micro-belt $(4.37 \mu \mathrm{A})$ in dark condition was very low. However, the photodetectors showed much higher photosensitivity at different incident light densities. It was obvious that the photocurrent increased significantly with increasing light intensity. It could be attributed to the change in photo-generated carriers. Under light irradiation, the $4 p$ orbital electrons of Ge and Se absorbed photon energy forming photo-generated carriers. The 
voltage bias then drove the photo-generated carriers to migrate along the direction of electric field and produced a photocurrent. The higher the voltage, the faster the carriers move. The higher the light power, the larger the quantity of the carrier. Thus, the photocurrent is positively correlated with the voltage bias or the light power. It was obvious that both photodetectors based on the single GeSe micro-sheet and the single GeSe micro-belt have a significant light response to the near infrared light $(980 \mathrm{~nm})$ and could output a signal with high ON/OFF ratios. Furthermore, the GeSe micro-belt-based photodetector presented higher dark current $(4.37 \mu \mathrm{A})$ and photocurrent $(44.6 \mu \mathrm{A})$ than those of GeSe micro-sheet $(1.17 \mu \mathrm{A}$ and $25.7 \mu \mathrm{A}$ ), but those should be attributed different internal mechanisms. On the one hand, high carrier concentration makes the GeSe micro-belt-based photodetector exhibit a high dark current. On the other hand, the smaller bandgap makes the micro-belts to get the larger photocurrent than the micro-sheets at $-7 \mathrm{~V}$. When light is radiated on the surface of the GeSe micro-belt-based photodetector, more energy can be absorbed and more photocarriers can be generated across a relatively narrow band gap. Then, a higher photocurrent can be produced in light radiated GeSe micro-belt photodetector. Figure S3 (Supporting Information) shows the I-V curves of the photodetector based on the single GeSe micro-sheets and the single GeSe micro-belts under dark, and illumination with different light intensities using $532 \mathrm{~nm}$ and $1319 \mathrm{~nm}$ laser as illumination sources, which also presented the significant light response to $532 \mathrm{~nm}$ and $1319 \mathrm{~nm}$.

Photoresponsivity $\left(R_{\lambda}\right)$, external quantum efficiency (EQE) and specific detectivity (D*) are important parameters to evaluate the photo-detecting performance. $R_{\lambda}$ can be defined as photocurrent generated per unit power of specific wavelength of incident light on the area. $\mathrm{EQE}$ is the number of electrons generated per incident photon of laser at specific wavelength. $\mathrm{D}^{*}$ is used to describe the sensitivity of the photodetector. These performances can be determined by formula (1), (2) and (3):

$R_{\lambda}=\left(I_{p h}-I_{d}\right) /(P \cdot S)$ 
$\mathrm{EQE}=\left(h \cdot c \cdot R_{\lambda}\right) /(e \cdot \lambda)$

$\mathrm{D}^{*}=R_{\lambda} \cdot S^{1 / 2} /\left(2 \cdot \mathrm{e} \cdot I_{d}\right)^{1 / 2}$

where, $I_{p h}$ is the current under the excitation of laser and $I_{d}$ is current without excitation in the dark; $S$ is the illuminated area of the GeSe micro-structure; $P$ is the power of the light; $c$ is the light speed; $h$ is the Planck's constant. Table 1 summarizes the $R_{\lambda}, \mathrm{EQE}$ and $\mathrm{D}^{*}$ of the photodetectors based on the single GeSe micro-sheet and the single GeSe micro-belt. Figure $7 \mathrm{a}$ and $\mathrm{b}$ show the dependence of $R_{\lambda}$ on the power of the light and the bias voltage for the photodetectors based on the single GeSe micro-sheet and the single GeSe micro-belt. The $R_{\lambda}$ increased with increasing the bias voltage and decreasing the power of the light. Under irradiation at $532 \mathrm{~nm}$ and bias voltage at $9 \mathrm{~V}$, the $R_{\lambda}$ reached up to $734 \mathrm{~A} / \mathrm{W}$ (single GeSe micro-sheet) and $5562 \mathrm{~A} / \mathrm{W}$ (single GeSe micro-sheet). The $R_{\lambda}$ of the photodetectors based on the single GeSe micro-belts was also as high as $1546 \mathrm{~A} / \mathrm{W}$ even under $980 \mathrm{~nm}$. At lower frequencies of infrared regime, in general, the energy of the single photon is too low to produce photogenerated carriers. Due to much higher carrier concentration (Table S1), however, the GeSe micro-belts generated more photo-generated carriers than GeSe microsheets. As a result, the $R_{\lambda}$ of the photodetector based on the single GeSe micro-belts could be calculated to be about 8 times $(980 \mathrm{~nm})$ and 92 times $(1319 \mathrm{~nm})$ as much as that of the photodetector based on the single GeSe micro-sheet. Moreover, the higher EQE and D* values (Table 1) also confirmed the excellent detection performance of the photodetector based on the single GeSe micro-belt.

The photodetectors based on the single GeSe micro-sheet and the single GeSe micro-belt also presented a fast photoresponse time in NIR. Figure $8 \mathrm{a}$ and $\mathrm{b}$ show the time-resolved photoresponse of the photodetectors based on the single GeSe micro-sheet and the single GeSe micro-belt under $980 \mathrm{~nm}$ laser illumination. When the applied voltage was $5 \mathrm{~V}$ (with an incident light density of $106.2 \mathrm{~mW} \cdot \mathrm{cm}^{-2}$ ), the current of the photodetector based on the single GeSe micro-belt dramatically increases from $2.43 \mu \mathrm{A}$ (OFF state, dark condition) to $11.29 \mu \mathrm{A}$ 
(ON state, light illumination). The light response remained the same after multiple cycles, demonstrating the excellent stability of the photodetectors based on the single GeSe microsheet and the single GeSe micro-belt. To extract the exact response time, one of the response periods in Figure 8a and $\mathrm{b}$ were magnified, as shown in Figure 8c and $\mathrm{d}$. In the time domain, the speed of a photodetector was often characterized by the response time ( $\tau_{\text {rise }}$, the time interval for the response to rise from $10 \%$ to $90 \%$ of its peak value) and the recovery time ( $\tau_{\text {decay }}$, the time interval for the response to decay from $90 \%$ to $10 \%$ of its peak value). The much shorter response time $(0.027 \mathrm{~s})$ and recovery time $(0.029 \mathrm{~s})$ of the photodetector based on the single GeSe micro-belt could be observed in comparison with the photodetector based on the single GeSe micro-sheet $\left(\tau_{\text {rise }}=0.2 \mathrm{~s}, \tau_{\text {decay }}=0.117 \mathrm{~s}\right)$.

Table 2 summarizes the key parameters of our photodetectors based on the single GeSe micro-sheet and the single GeSe micro-belt, compared to various low dimension materialsbased photodetectors reported in the literature. Both photodetectors based on the single GeSe micro-sheet and the single GeSe micro-belt demonstrated considerable or much better photoelectric performance, especially for the photodetectors based on the single GeSe microbelt. For the visible light, the $R_{\lambda}$ of the photodetectors based on the single GeSe micro-belt $(5562 \mathrm{~A} / \mathrm{W})$ was higher than $\mathrm{MoS}_{2}$ CVD layer $(2200 \mathrm{~A} / \mathrm{W})^{[30]}$. For the NIR light, the $R_{\lambda}$ at $980 \mathrm{~nm}(1546 \mathrm{~A} / \mathrm{W})$ is much higher than that $(6.21 \mathrm{~A} / \mathrm{W})$ of $\mathrm{Sn}$ doped $\mathrm{Sb}_{2} \mathrm{Se}_{3}$-based photodetector at $850 \mathrm{~nm}{ }^{[38]}$. Moreover, the response time $(0.027 \mathrm{~s})$ of the photodetector based on the single GeSe micro-belt was the faster than GaS-based photodetector $(0.03 \mathrm{~s})^{[35]}$, GaTebased photodetector $(0.054 \mathrm{~s})^{[36]}$, GeS-based photodetector $(0.11 \mathrm{~s})^{[19]}$ and $2 \mathrm{D}$ GeSe-based photodetector $(0.2 \mathrm{~s})^{[20]}$ or phototransistor $(0.28 \mathrm{~s})^{[21]}$. At the same time, both photodetectors based on the single GeSe micro-sheet and the single GeSe micro-belt also presented photoresponse at longer wavelength $(1319 \mathrm{~nm})$. These excellent performances make the photodetector based on the single GeSe micro-sheet (belt) a promising material/concept for designing highly sensitive photodetectors, especially in NIR photodetection. 


\section{Conclusion}

In conclusion, the GeSe micro-sheets and the GeSe micro-belts were synthesized by one-pot wet chemical method in 1-octadecene solvent and oleic acid solvent, respectively. The larger adsorption of oleic acid molecules on the (002) plane of the GeSe micro-belt promoted the growth along [010] direction. Owing to the limitation of carrier transport in one direction along [010], the GeSe micro-belts exhibited much higher carrier concentration and mobility $\left(31.2 \mathrm{~cm}^{2} \cdot \mathrm{V}^{-1} \cdot \mathrm{s}^{-1}, 1.601 \times 10^{14} \mathrm{~cm}^{-2}\right)$ than the GeSe micro-sheets $\left(7.75 \mathrm{~cm}^{2} \cdot \mathrm{V}^{-1} \cdot \mathrm{s}^{-1}\right.$, $2.447 \times 10^{12} \mathrm{~cm}^{-2}$ ). As a result, the photodetector based on the single GeSe micro-belt showed remarkable broadband response with a high responsivity $(5562 \mathrm{~A} / \mathrm{W}$ at $532 \mathrm{~nm}, 1546 \mathrm{~A} / \mathrm{W}$ at $980 \mathrm{~nm})$ and detectivity $\left(3.01 \times 10^{12}\right.$ Jones at $532 \mathrm{~nm}, 8.38 \times 10^{11}$ Jones at $\left.980 \mathrm{~nm}\right)$, short response/recovery times $(0.027 / 0.029$ s), and long-term durability. These excellent performances make the photodetector based on the single GeSe micro-belt very interesting as highly efficient photodetectors, especially in NIR region.

\section{Experimental Section}

Materials: Germanium (VI) iodide ( $\mathrm{GeI}_{4}, 99.999 \%$, Sigma Aldrich), selenium dioxide ( $\mathrm{SeO}_{2}$, Alfa Aesar, 99.4\%), 1-octadecene (Aladdin, 90\%), 1-Hexadecylamine (Aladdin, 90\%) and oleic acid (Sigma-Aldrich, 90\%). Cyclohexane (99.5\%) and absolute ethyl alcohol (99.7\%) were purchased from Sinopharm Chemical Reagent Co., Ltd. All reagents were used for the synthesis without further purification.

Synthesis of GeSe micro-sheets: For the synthesis of GeSe micro-sheets, $\mathrm{GeI}_{4}(0.29 \mathrm{~g})$, $\mathrm{SeO}_{2}(0.05548 \mathrm{~g})$, 1-octadecene $(6 \mathrm{ml})$ and 1-Hexadecylamine $(3 \mathrm{~g})$ were added into a threeneck flask. The mixture was heated at $120{ }^{\circ} \mathrm{C}$ for about 15 minutes under vacuum at the stirring speed of $400 \mathrm{r} / \mathrm{min}$ until the selenium dioxide was completely dissolved in 1octadecene (solution became transparent brown). And then Ar gas was introduced into the 
flask and the solution was heated to $320{ }^{\circ} \mathrm{C}$. The solution was stirred at $320{ }^{\circ} \mathrm{C}$ for $6 \mathrm{~h}$ and then cooled down to room temperature. The obtained solid GeSe micro-sheets were precipitated using an ethanol/cyclohexane mixture (ethanol: cyclohexane $=1: 1$ ) and then were collected by centrifugation, washed with ethanol for several times, and finally dried at $60^{\circ} \mathrm{C}$.

Synthesis of GeSe micro-belts: The preparation process is same as for micro-sheets except that the 1-octadecene as solvent was replaced by oleic acid.

Hall effect measurement: the obtained GeSe micro-sheets or micro-belts powders were put in a round mould for pressing. Then the powder-filled mould was put into the tablet press and the GeSe powders were punched into a round film at a pressure of $40 \mathrm{MPa}$ for about 30 seconds. After coating four Ag electrodes on the film, the GeSe micro-sheets or micro-belt films for Hall effect measurement were fabricated. Then the film was put into the device (BID-RAD HL5500PC) for Hall effect measurement.

Fabrication of the photodetector devices: Two photodetectors were fabricated from microsheets and micro-belts, respectively. The as-synthesized GeSe micro-sheets (micro-belts) were ultrasonically dispersed in ethanol solution. Subsequently, a capillary was used to drop the ethanol solution containing GeSe micro-sheets (micro-belts) on a clean silicon wafer. Then some micro-sheets (micro-belts) were picked up by PDMS (Polydimethylsiloxane) under the microscope. After that we selected a single micro-sheet (micro-belt) stuck under PDMS, aligned it with the gold electrode on $\mathrm{SiO}_{2} / \mathrm{Si}$, and then moved PDMS down to connect the selected single micro-sheet (single micro-belt) to the gold electrode. After heating at $180{ }^{\circ} \mathrm{C}$ for 5 minutes, PDMS was lifted and single micro-sheet (single micro-belt) stayed on the electrode. Figure S4 shows fabrication schematic diagram of the photodetector.

Characterization: X-ray diffraction (XRD) measurements were performed on Daojin XRD6000, using $\mathrm{Cu} \mathrm{K \alpha}$ radiation $(\lambda=1.54 \AA)$. Transmission electron microscopy (TEM) images and high resolution TEM (HRTEM) images were obtained from Titan ChemiSTEM. Samples were prepared by dropping a diluted GeSe sheets or belts solution onto carbon coated copper 
grids. S-4800 scanning electron microscope (SEM) and MultiMode atomic force microscope (AFM) were used to observe the morphology of the GeSe micro-sheets and micro-belts. The electrical properties of GeSe micro-sheets and micro-belts were investigated by the Hall measurement with (BID-RAD HL5500PC). The reflectance spectra were acquired using a Hitachi U4100 spectrophotometer. The photoresponse performance of the devices were measured by a Keithley 4200 SCS. The $532 \mathrm{~nm}, 980 \mathrm{~nm}$ and $1319 \mathrm{~nm}$ laser diodes were used as the light source for the photocurrent measurements. FTIR spectra was measured by Nicolet iS10 FT-IR spectrometer.

\section{Supporting Information}

Supporting Information is available from the Wiley Online Library or from the author.

\section{Acknowledgements}

The authors gratefully acknowledge the financial support from the Nature Science Foundation of China (No. 61674127; 61874094), State Key Laboratory of Silicon Materials, ZJ-NSF (LZ17F040001), the National Key Basic Research Program of China (2016YFA0200204, 2016YFA0301204), Zhejiang Key Laboratory for Advanced Microelectronic Intelligent Systems and Applications, and ZJU Micro-Nano Fabrication Center and. X. Qiao acknowledges financial support from China Scholarship Council (CSC).

Received: ((will be filled in by the editorial staff))

Revised: ((will be filled in by the editorial staff))

References Published online: ((will be filled in by the editorial staff))

[1] Iijima, Sumio, Nature 1991, 354, 56-58.

[2] Y. Zhang, H. Ago, J. Liu, M. Yumura, K. Uchida, S. Ohshima, S. Iijima, J. Zhu, X. Zhang, Journal of Crystal Growth 2004, 264, 363-368.

[3] S. H. Choi, K. L. Wang, M. S. Leung, G. W. Stupian, N. Presser, B. A. Morgan, R. E. Robertson, M. Abraham, E. E. King, M. B. Tueling, S. W. Chung, J. R. Heath, S. L. Cho, J. B. Ketterson, Journal of Vacuum Science \& Technology A: Vacuum, Surfaces, and Films 2000, $18,1326-1328$.

[4] S. W. Chung, J. Y. Yu, J. R. Heath, Applied Physics Letters 2000, 76, 2068-2070. 
[5] H. Kind, H. Yan, B. Messer, M. Law. P. Yang, Advanced Materials 2002,14,158-160.

[6] C. Soci, A. Zhang, B. Xiang, S. A. Dayeh, D. P. Aplin, J. Park, X. Y. Bao, Y. H. Lo, D. Wang, Nano Lett. 2007, 7, 1003-1009.

[7] K. S. Novoselov, D. Jiang, F. Schedin, T. J. Booth, V. V. Khotkevich, S. V. Morozov, A. K. Geim, Proceedings of the National Academy of Sciences of the United States of America 2005, 102, 10451-10453.

[8] C. Lee, X. Wei, J. W. Kysar, J. Hone, Science 2008, 321, 385-388.

[9] A. K. Geim, K. S. Novoselov, Nature Materials 2007, 6, 183-191.

[10] B. Radisavljevic, A. Radenovic, J. Brivio, V. Giacometti, A. Kis, Nature Nanotechnology 2011, 6, 147-150.

[11] K. F. Mak, C. Lee, J. Hone, J. Shan, T. F. Heinz, Physical Review Letters 2010, 105, 136805.

[12] F. Xia, H. Wang, D. Xiao, M. Dubey, A. Ramasubramaniam, Nature Photonics 2014, 8, 899-907.

[13] L. K. Li, Y. J. Yu, G. J. Ye, Q. Q. Ge, X. D. Ou, H. Wu, D. L. Feng, X. H. Chen, Y. B. Zhang, Nature Nanotechnology 2014 9, 372.

[14] J. D. Wood, S. A. Well, D. Jariwala, K. S. Chen, E. K. Cho, V. K. Sangwan. X. L. Liu, L. J. Lauhon, T. J. Marks, M. C. Hersam, Nano Letters 2014,14, 6964-6970.

[15] Y. Hu, S. Zhang, S. Sun, M. Xie, B. Cai, H. Zeng, Applied Physics Letters 2015, 107, 122107.

[16] Y. Kim, I.-H. Choi, Journal of the Korean Physical Society 2018, 72, 238-242.

[17] Y. Ye, Q. Guo, X. Liu, C. Liu, J. Wang, Y. Liu, J. Qiu, Chemistry of Materials 2017, 29, $8361-8368$

[18] B. Mukherjee, Y. Cai, H. R. Tan, Y. P. Feng, E. S. Tok, C. H. Sow, ACS Appl. Mater. Interfaces 2013, 5, 9594-9604. 
[19] P. Ramasamy, D. Kwak, D. H. Lim, H. S. Ra, J. S. Lee, Journal of Materials Chemistry C 2016, 4, 479-485.

[20] D. Ma, J. Zhao, R. Wang, C. Xing, Z. Li, W. Huang, X. Jiang, Z. Guo, Z. Luo, Y. Li, J. Li, S. Luo, Y. Zhang, H. Zhang, ACS Appl. Mater. Interfaces 2019, 11, 4278-4287

[21] X. Zhou, X. Hu, B. Jin, J. Yu, K. Liu, H. Li, T. Zhai, Advanced Science (Weinheim, Germany) 2018, 5, 1800478

[22] L. Shi, Y. Li, Y. Dai, ChemPlusChem 2015, 80, 630-634.

[23] D. J. Xue, J. Tan, J. S. Hu, W. Hu, Y. G. Guo, L. J. Wan, Advanced Materials 2012, 24, $4528-4533$

[24] L. Shi, Y. Dai, Materials Letters 2014, 116, 123-126.

[25] D. Vaughn, D. Sun, S. M. Levin, A. J. Biacchi, T. S. Mayer, R. E. Schaak, Chemistry of Materials 2012, 24, 3643-3649.

[26] D. D. Vaughn, R. J. Patel, M. A. Hickner, R. E. Schaak, Journal of the American Chemical Society 2010, 132, 15170-15172.

[27] N. Shukla, C. Liu, P. M. Jones and D. Weller, Journal of Magnetism Magnetic Materials 2003, 206, 178-184.

[28] H. Zhao, Y. Mao, X. Mao, X. Shi, C. Xu, C. Wang, S. Zhang, D. Zhou, Advanced Functional Materials 2018, 28, 1704885.

[29] G. K. Solanki, M. P. Deshpande, M. K. Agarwal, P. D. Patel, Journal of Materials Science Letters 2003,22,985-987.

[30] W. Zhang, J. K. Huang, C. H. Chen, Y. H. Chang, Y. J. Cheng, L. J. Li, Advanced materials 2013, 25, 3456-3461.

[31] O. Lopez-Sanchez, D. Lembke, M. Kayci, A. Radenovic, A. Kis, Nat Nanotechnol 2013, $8,497-501$ 
[32] X. Wang, P. Wang, J. Wang, W. Hu, X. Zhou, N. Guo, H. Huang, S. Sun, H. Shen, T. Lin, M. Tang, L. Liao, A. Jiang, J. Sun, X. Meng, X. Chen, W. Lu, J. Chu, Advanced Materials 2015, 27, 6575-6581.

[33] M. Buscema, D. J. Groenendijk, S. I. Blanter, G. A. Steele, H. S. Van Der Zant, A. Castellanos-Gomez, Nano Letters 2014, 14, 3347-3352.

[34] Y. Xu, J. Yuan, L. Fei, X. Wang, Q. Bao, Y. Wang, K. Zhang, Y. Zhang, Small 2016, 12, 5000-5007.

[35] P. Hu, L. Wang, M. Yoon, J. Zhang, W. Feng, X. Wang, Z. Wen, J. C. Idrobo, Y. Miyamoto, D. B. Geohegan, K. Xiao, Nano Letters 2013, 13, 1649-1654.

[36] Z. Wang, M. Safdar, M. Mirza, K. Xu, Q. Wang, Y. Huang, F. Wang, X. Zhan, J. He, Nanoscale 2015, 7, 7252-7258.

[37] R. K. Ulaganathan, Y. Y. Lu, C. J. Kuo, S. R. Tamalampudi, R. Sankar, K. M. Boopathi, A. Anand, K. Yadav, R. J. Mathew, C. R. Liu, F. C. Chou, Y. T. Chen, Nanoscale 2016, 8, 2284-2292.

[38] S. Chen, K. Shehzad, X. Qiao, X. Luo, X. Liu, Y. Zhang, X. Zhang, Y. Xu, X. Fan, Journal of Materials Chemistry C 2018, 6, 11078-11085.

[39] X. Wang, Y. Li, L. Huang, X. W. Jiang, L. Jiang, H. Dong, Z. Wei, J. Li, W. Hu, Journal of American Chemical Society 2017, 139, 14976-14982. 

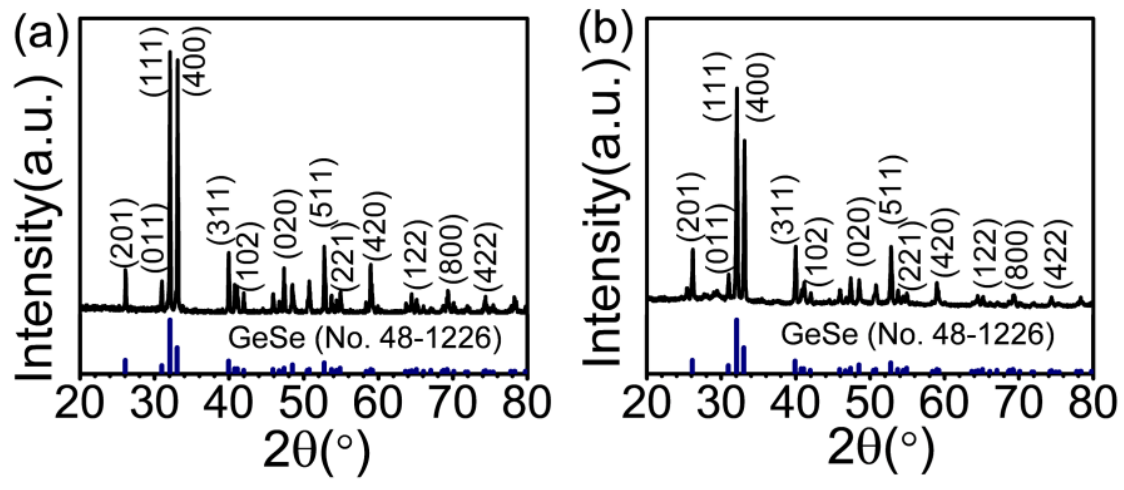

Figure 1. XRD patterns of (a) GeSe micro-sheets and (b) GeSe micro-belts.

(a)

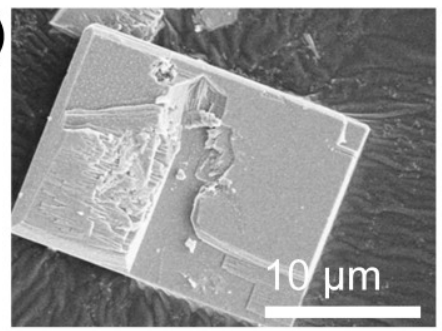

(d)

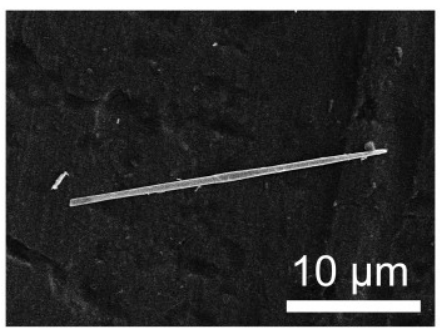

(b)

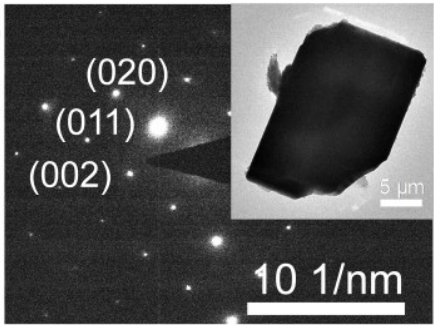

(e)

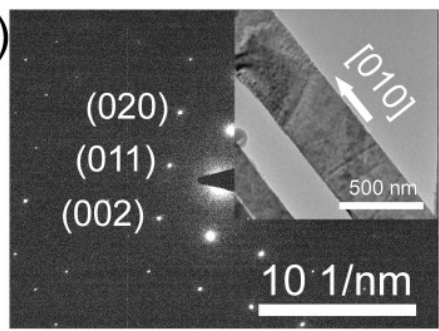

(c)

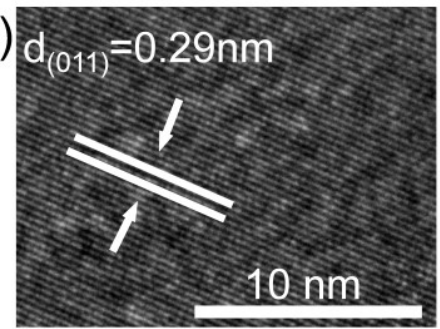

(f)

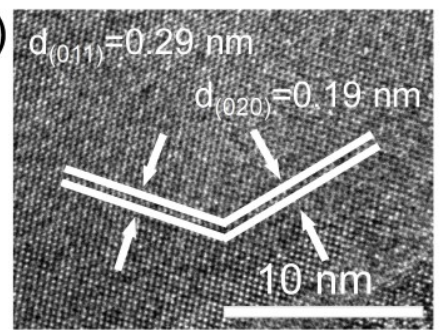

Figure 2. SEM images of (a) GeSe micro-sheet and (d) GeSe micro-belt. SAED patterns of

(b) GeSe micro-sheet and (e) GeSe micro-belt. HRTEM images of (c) GeSe micro-sheet and (f) GeSe micro-belt. 
(a)

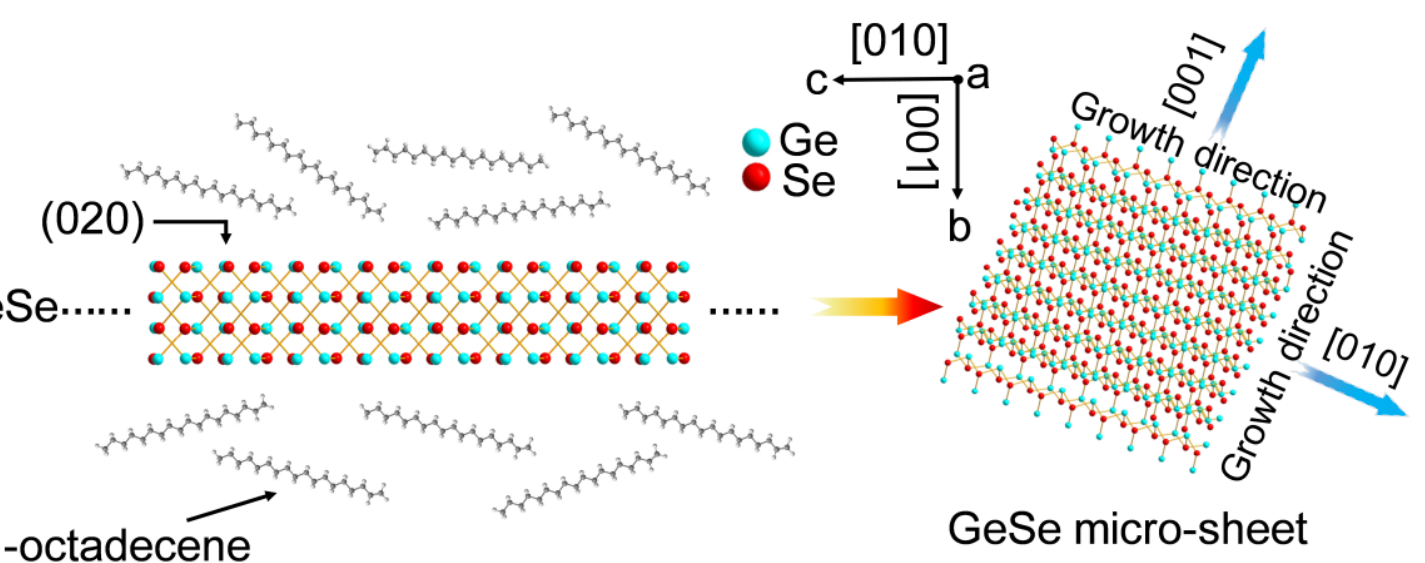

(b)
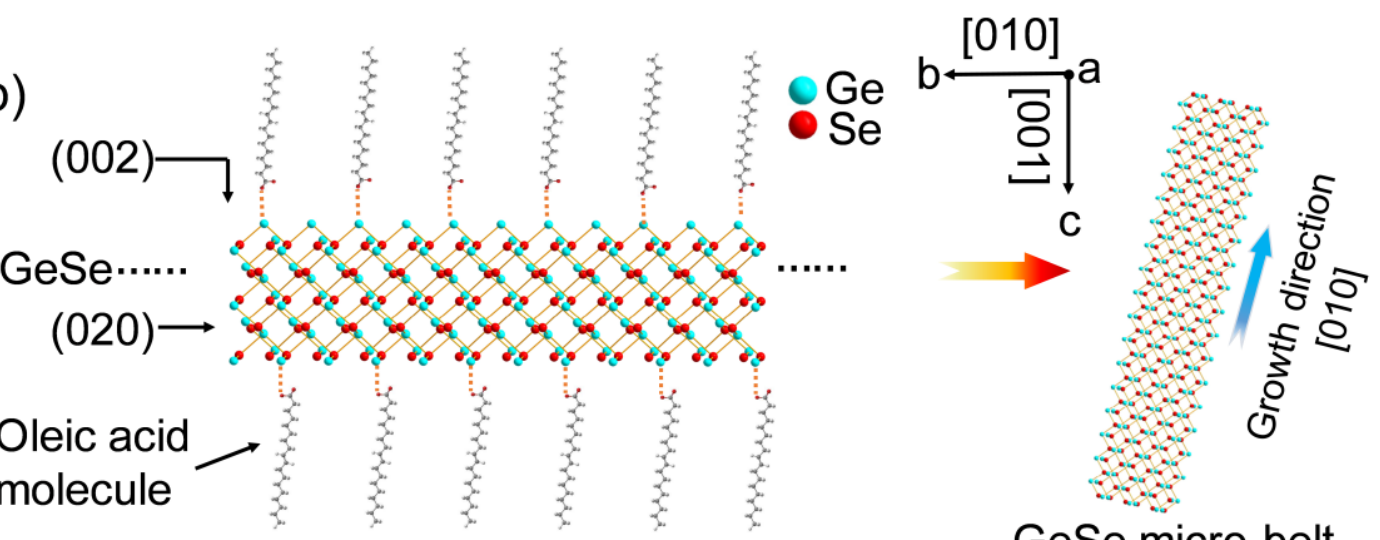

GeSe micro-belt

Figure 3. Formation mechanism of (a) GeSe micro-sheets and (b) micro-belts.

(a)

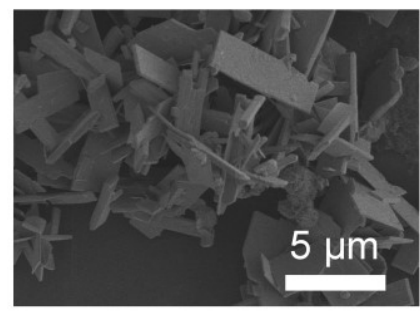

(d)

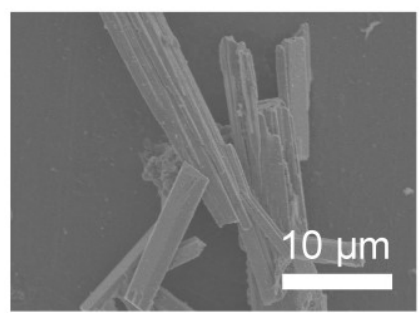

(b)

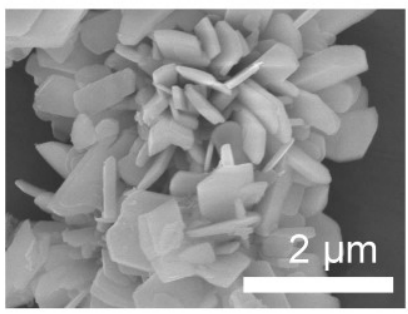

(e)

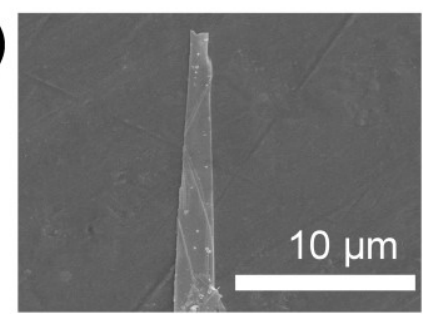

(c)

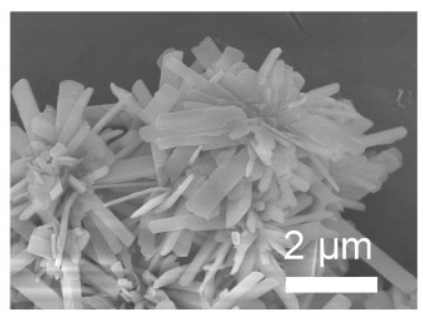

(f)

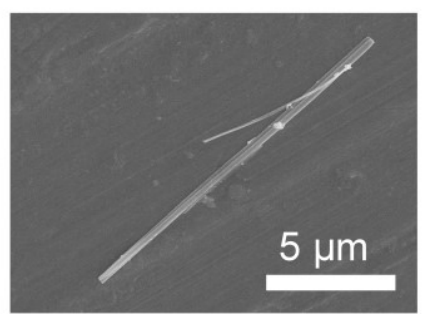

Figure 4. GeSe morphologies with different oleic acid/1-octadecene ratios: (a) 0:100; (b)

$$
\text { 20:80; (c) 40:60; (d) 60:40; (e) 80:20; (f) 100:0. }
$$



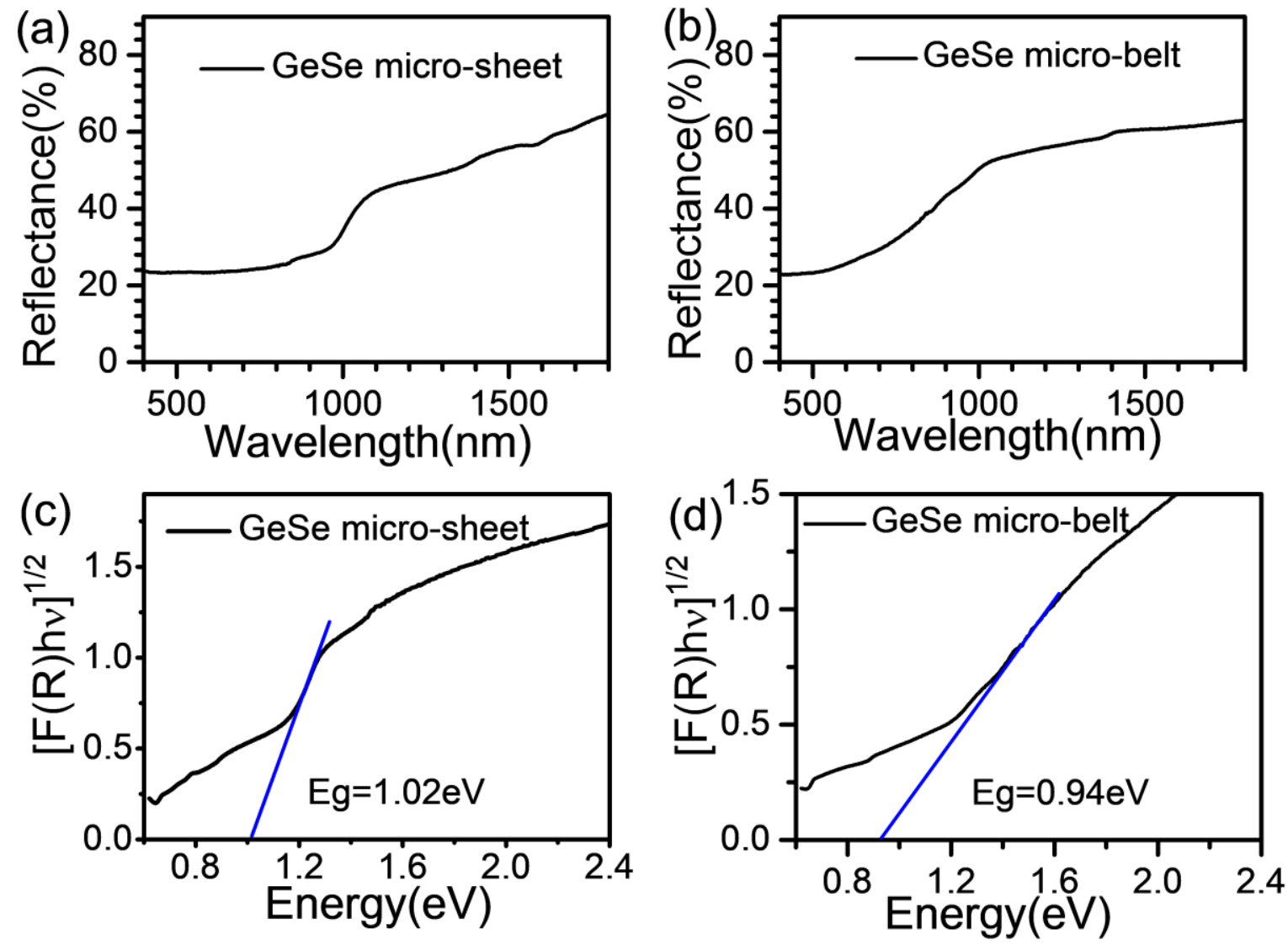

Figure 5. Reflectance spectra of (a) GeSe micro-sheets and (b) micro-belts. Indirect bandgap by KM function of (c) GeSe micro-sheets and (d) micro-belts.

(a)
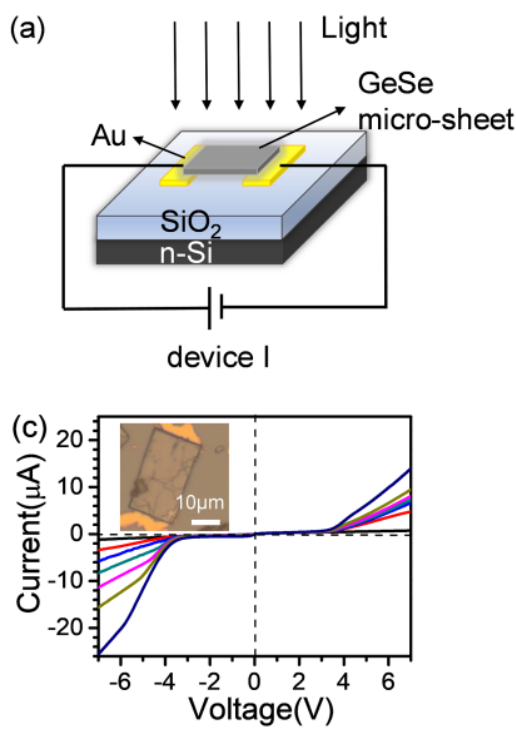

(b)
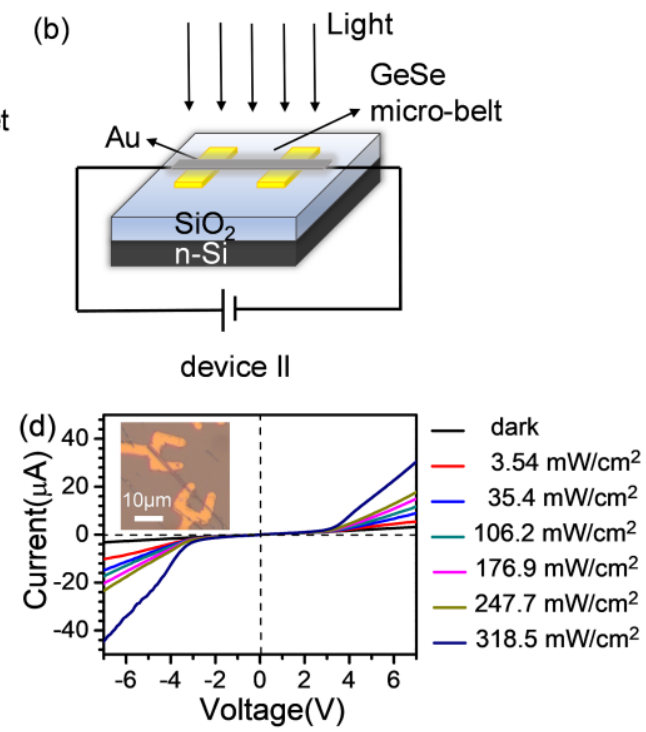
Figure 6. Diagrams of photodetectors fabricated by (a) single micro-sheet and (b) single micro-belt, respectively. I-V curves of the photodetectors based on (c) single GeSe microsheet and (d) single micro-belt under $980 \mathrm{~nm}$. Inset: Optical images of the single GeSe microsheet (single micro-belt) on the Au electrodes.
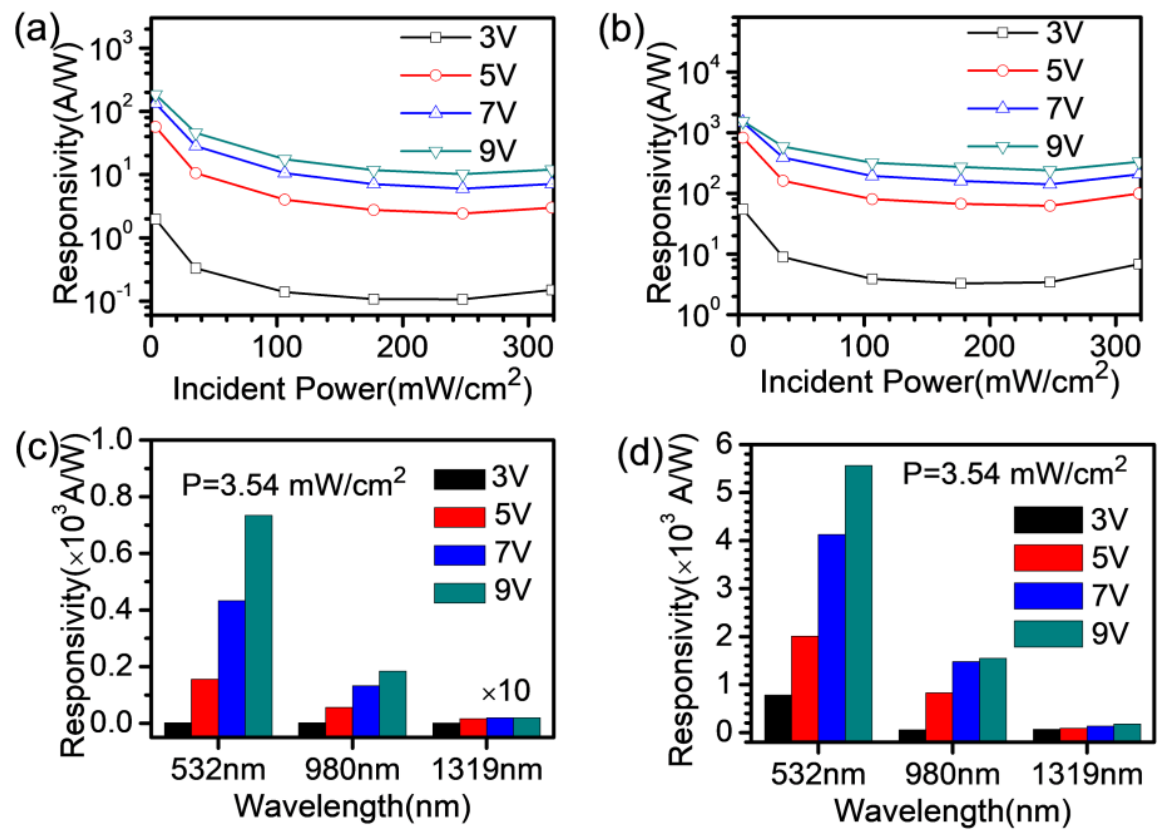

Figure 7. Power-dependence responsivity $\left(R_{\lambda}\right)$ of the photodetectors based on (a) single GeSe micro-sheet and (b) single GeSe micro-belt at different bias voltage (under $980 \mathrm{~nm}$ ). Wavelength-dependent responsivity $\left(R_{\lambda}\right)$ of the photodetectors based on (c) single GeSe micro-sheets and (d) single GeSe micro-belts at different bias voltage. 

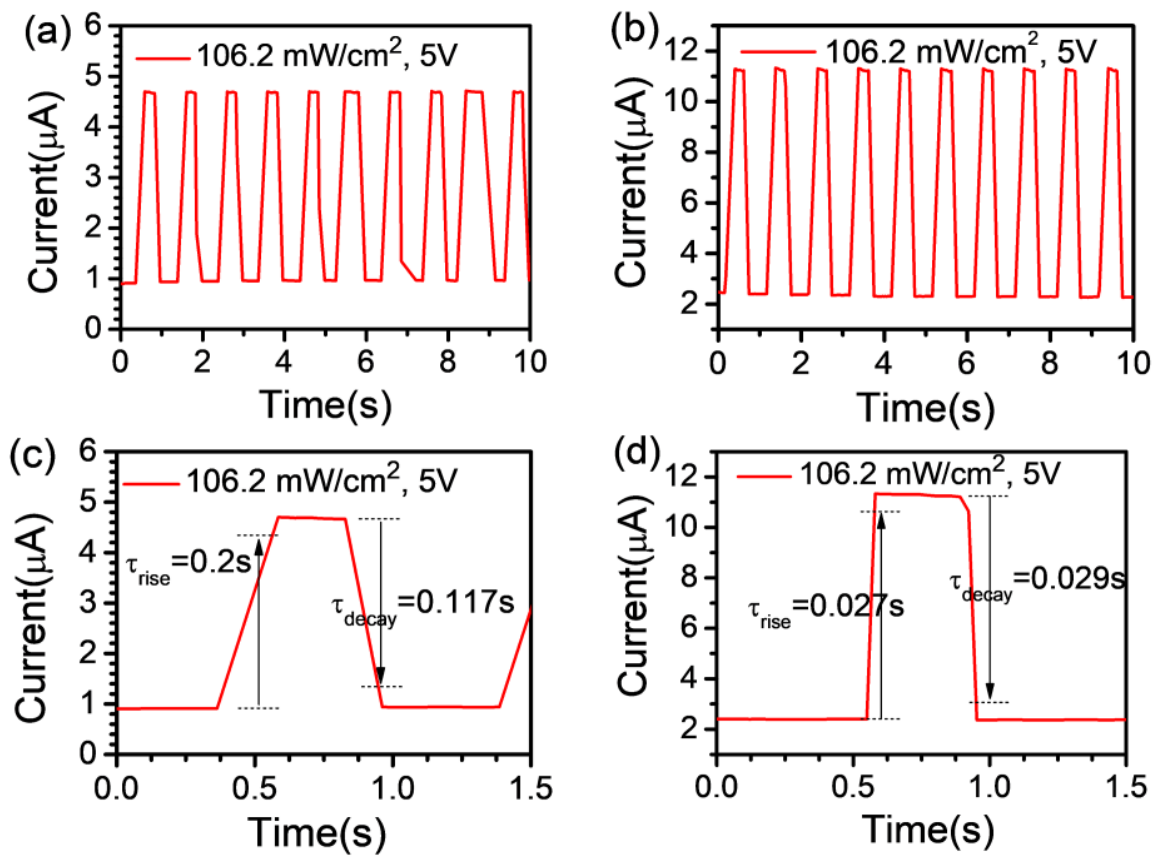

Figure 8. Time-resolved photoresponse $(980 \mathrm{~nm})$ of the photodetectors based on (a) single GeSe micro-sheet and (b) single GeSe micro-belt at the bias of $5 \mathrm{~V}$ with an incident light density of $106.2 \mathrm{~mW} \mathrm{~cm}^{-2}$. Response time and the recovery time of the photodetectors based on (c) single GeSe micro-sheets and (d) single GeSe micro-belts at a bias of $5 \mathrm{~V}$. 
Table 1. $R_{\lambda}$, EQE and $\mathrm{D}^{*}$ of the photodetectors based on single GeSe micro-sheet and single GeSe micro-belt under $532 \mathrm{~nm}, 980 \mathrm{~nm}$ and $1319 \mathrm{~nm}$.

\begin{tabular}{rrrrrrrr}
\hline & \multicolumn{3}{c}{ Micro-sheet } & & \multicolumn{3}{c}{ Micro-belt } \\
\cline { 2 - 4 } \cline { 6 - 8 } & $532 \mathrm{~nm}$ & $980 \mathrm{~nm}$ & $1319 \mathrm{~nm}$ & & $532 \mathrm{~nm}$ & $980 \mathrm{~nm}$ & $1319 \mathrm{~nm}$ \\
\hline $\mathrm{R}_{\lambda}(\mathrm{A} / \mathrm{W})$ & 734 & 184 & 1.94 & & 5562 & 1546 & 179 \\
$\mathrm{EQE}(\%)$ & $1.72 \times 10^{5}$ & $2.33 \times 10^{4}$ & $1.82 \times 10^{2}$ & & $1.30 \times 10^{6}$ & $1.96 \times 10^{5}$ & $1.68 \times 10^{4}$ \\
$\mathrm{D}^{*}$ (Jones) & $3.20 \times 10^{12}$ & $8.02 \times 10^{11}$ & $8.46 \times 10^{9}$ & & $3.01 \times 10^{12}$ & $8.38 \times 10^{11}$ & $9.71 \times 10^{10}$ \\
\hline
\end{tabular}

Table 2. Comparison of key parameters between this work and various low dimension materials-based photodetectors.

\begin{tabular}{rrrrrr}
\hline Materials & $\begin{array}{r}\text { Wavelength } \\
(\mathrm{nm})\end{array}$ & $\begin{array}{r}R_{\lambda} \\
(\mathrm{A} / \mathrm{W})\end{array}$ & $\begin{array}{r}\text { EQE } \\
(\%)\end{array}$ & $\begin{array}{r}\text { Response time } \\
(\mathrm{s})\end{array}$ & Reference \\
\hline $\mathrm{MoS}_{2}$ & 473 & 2200 & & 220 & 30 \\
$\mathrm{MoS}_{2}$ & 561 & 880 & & 0.6 & 31 \\
$\mathrm{MoS}_{2}$ & 635 & 2570 & & 0.0018 & 32 \\
Black phosphorus & 640 & $4.8 \times 10^{-3}$ & & 0.001 & 33 \\
Sn-doped black phosphorus & 635 & 15.33 & 2.993 & 0.3 & 34 \\
$\mathrm{GaS}$ & 254 & 4.2 & $2.05 \times 10^{3}$ & 0.03 & 35 \\
$\mathrm{GaTe}$ & 473 & 0.03 & 8 & 0.054 & 36 \\
$\mathrm{GeS}$ & 633 & 206 & $4 \times 10^{4}$ & & 37 \\
$\mathrm{GeS}$ & 405 & 173 & $5.32 \times 10^{4}$ & 0.11 & 19 \\
$\mathrm{Sb}_{2} \mathrm{Se} \mathrm{H}_{3}$ & 850 & 6.21 & 906 & 0.04 & 38 \\
$\mathrm{GeSe}$ & 405 & 870 & $2.67 \times 10^{5}$ & 0.15 & 19 \\
$\mathrm{GeSe}$ & 808 & 3.5 & 537 & 0.1 & 18 \\
$\mathrm{GeSe}($ nanosheet) & 350 & $2.3 \times 10^{-3}$ & 816 & 0.2 & 20 \\
$\mathrm{GeSe}($ nanosheet) & 532 & $1.6 \times 10^{5}$ & $3.9 \times 10^{7}$ & 0.28 & 39 \\
$\mathrm{GeSe}($ nanoflake) & 532 & 4.25 & & & \\
GeSe (sheet) & 532 & 734 & $1.72 \times 10^{5}$ & 0.194 & This work \\
& 980 & 184 & $2.33 \times 10^{4}$ & 0.117 & \\
GeSe (belt) & 1319 & 1.94 & $1.82 \times 10^{2}$ & 0.138 & This work \\
& 532 & 5562 & $1.30 \times 10^{6}$ & 0.031 & \\
\hline
\end{tabular}


Table of contents: GeSe micro-sheets and micro-belts were synthesized by a chemical orientation growth method in 1-octadecene solvent and oleic acid solvent, respectively. The photodetectors based on single micro-belt showed a broadband spectral photoresponse with high responsivity $(5562 \mathrm{~A} / \mathrm{W})$ and detectivity $\left(3.01 \times 10^{12}\right.$ Jones $)$. It demonstrates significant application potential in the visible and NIR photodetection.

Keyword: GeSe; photodetectors; micro-sheet; micro-belt; photoresponsivity

Feiyu Zhao, ${ }^{\mathrm{a}}$ Xue Luo, ${ }^{\mathrm{a}}$ Lixiang Liu, ${ }^{\mathrm{b}}$ Jianhang Lv, ${ }^{\mathrm{b}}$ Xianping Fan, ${ }^{\text {* }}$ Xvsheng Qiao, ${ }^{\text {* }}$ Yang $\mathrm{Xu},{ }^{\mathrm{a}, \mathrm{b}^{*}}$ Lorenz Kienle, ${ }^{\mathrm{c}}$ Xianghua Zhang, ${ }^{\mathrm{d}}$ Guodong Qian ${ }^{\mathrm{a}}$

Title: Visible-NIR Photodetectors Based on Low Dimensional GeSe Micro-crystals: Designed Morphology and Improved Photoresponsivity 


\section{ToC figure}

Surface adsorption of the ligands induces GeSe to grow into micro-belts with high photoelectric performance.
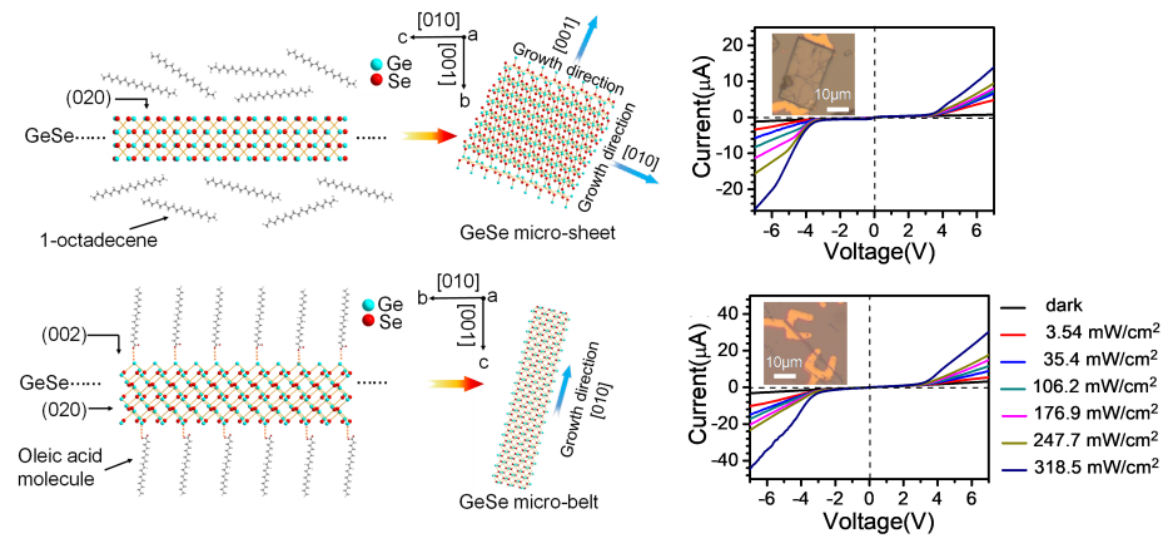